\title{
A MEMS Based Visible-NIR Fourier Transform Microspectrometer
}

\author{
C. Ataman ${ }^{1}$, H. Urey ${ }^{1}$, S.O. Isikman ${ }^{1}$, and A. Wolter ${ }^{2}$ \\ ${ }^{1}$ Optical Microsystems Laboratory, Department of Electrical Engineering, Koc University \\ Rumeli Feneri Yolu, 34450, Sariyer, Istanbul, TURKEY \\ Phone: +90-212-338-1474, E-mail: cataman@ku.edu.tr, hurey@ku.edu.tr \\ ${ }^{2}$ Fraunhofer Institute of Photonic Microsystems, Maria-Reiche-Str. 201109 Dresden, Germany \\ E-mail: alexander.wolter@ipms.fraunhofer.de
}

\begin{abstract}
Design, fabrication and characterization of a novel out-of-plane vertical comb-drive actuator based Fourier transform microspectrometer (FTS) is presented. The spectrometer utilizes resonant mode vertical comb actuators as a variable-depth diffraction grating and a single photodetector to monitor the 0th order of the diffraction pattern. The spectrum of the source illuminating the gratings is computed by Fourier transforming the 0 th order intensity as a function of the optical path difference. The vertical comb actuators have a travel range of $100 \mu \mathrm{m}$ under atmospheric pressure with $28 \mathrm{~V}$ excitation, which yields a theoretical spectral resolution of $0.5 \mathrm{~nm}$ in the visible and better than $5 \mathrm{~nm}$ in the telecom wavelengths.
\end{abstract}

Keywords: Comb actuation, Fourier transform spectroscopy, MEMS, diffraction grating.

\section{INTRODUCTION}

A lamellar grating interferometer (LGI) is a special type of Fourier transform spectrometer based on a diffraction grating operating in the $0^{\text {th }}$ order [1]. If multiple periods of a resonating vertical comb actuator are illuminated by a light beam, the light diffracts into several different orders equally spaced by an angle determined by the period of the fingers. The intensity at the center of the $0^{\text {th }}$ diffraction order depends solely on the spectral content of the illuminating light. Fourier transform of the $0^{\text {th }}$ order intensity as the optical path difference of the grating changes gives the spectrum of the diffracted beam. For high resolution spectrometry, the travel range of the movable fingers should be as high as possible. Due to the lack of a beam-splitter in an LGI very compact spectrometers with good performance can be realized.

A Lamellar Grating Interferometer based on out-of plane mode resonant comb actuators is presented. Out-ofplane resonant mode operation of the comb actuators allows for large deflections (high spectral resolution) while providing a large light collection mirror area compared to other microspectrometers reported in the literature [23]. Fabrication and operation principles of the device are simple. This minimizes the size, complexity, cost and design effort of a fully functional system (an operational spectrometer using this device requires integration with a simple photodetector and readout circuit). Only moving part is the resonating MEMS structure. With slight modifications in the device dimensions, spectrometers for very wide range of wavelengths can be realized.

Structural properties of the movable grating designed for this application is presented in section 2. Operation principle of the spectrometer system is described in section 3. Section 4 explores the theoretical and experimental performance analysis results for the system.

MEMS, MOEMS, and Micromachining II, edited by Hakan Ürey, Ayman El-Fatatry,

Proc. of SPIE Vol. 6186, 61860C, (2006)

0277-786X/06/\$15 $\cdot$ doi: $10.1117 / 12.664781$

Proc. of SPIE Vol. 6186 61860C-1 


\section{MOVABLE GRATING STRUCTURE}

\subsection{Device Layout}

The device is fabricated and packaged inside a LCC housing by Fraunhofer IPMS, Germany. In Figure 2, a picture of the packaged device can be seen. The core of the structure consists of two sets of electrostatic comb fingers that are simultaneously utilized as an actuator and a variable-depth diffraction grating. The comb fingers have a high fill-factor (70 $\mu \mathrm{m}$ finger width and $5 \mu \mathrm{m}$ gap) and are covered with a thin aluminum layer to enhance reflectivity; thus a high light efficiency is obtained. Movable comb fingers are attached to a $250 \mu \mathrm{m}$ wide $\mathrm{H}$ shaped backbone to increase the stability. This backbone is bonded to the fixed frame via four folded flexure beams, which provide low stiffness within a compact structure. The folded flexures which have varying crosssectional width to have uniform stress distribution and to provide low stiffness within a compact structure [4]. Low stiffness springs are essential to lower the resonance frequency and increase deflection. These effects decrease the required speed of the readout electronics and enhance spectral resolution, respectively. The flexures are placed at a $45^{\circ}$ angle to obtain a better mode separation between the fundamental and higher modes. The folded flexure arms get thinner in cross-section towards the middle of the beam, which helps uniform stress distribution within the flexure.

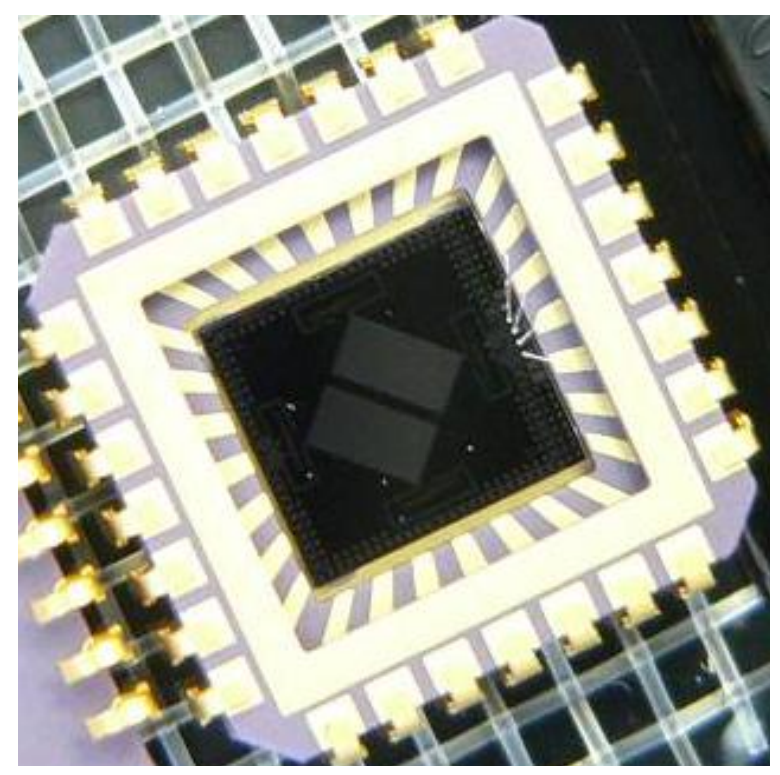

Figure 1: Packaged device

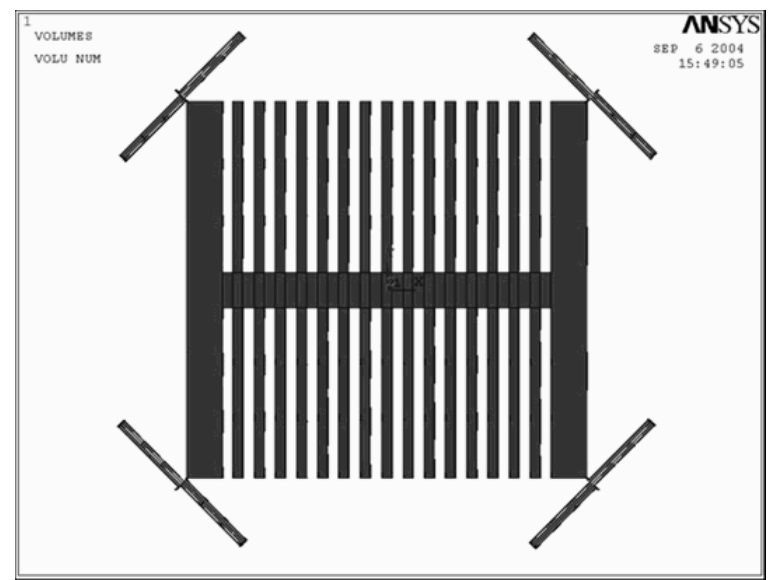

Figure 2: The ANSYS model of the spectrometer. The fixed comb fingers are not shown in the Figure. 
The spectrometer is fabricated on silicon-on-insulator (SOI) wafer, and the process is CMOS compatible. Details of similar process flows were given elsewhere [5]. A cross-section drawing showing the fabricated layers is given in Figure 3.

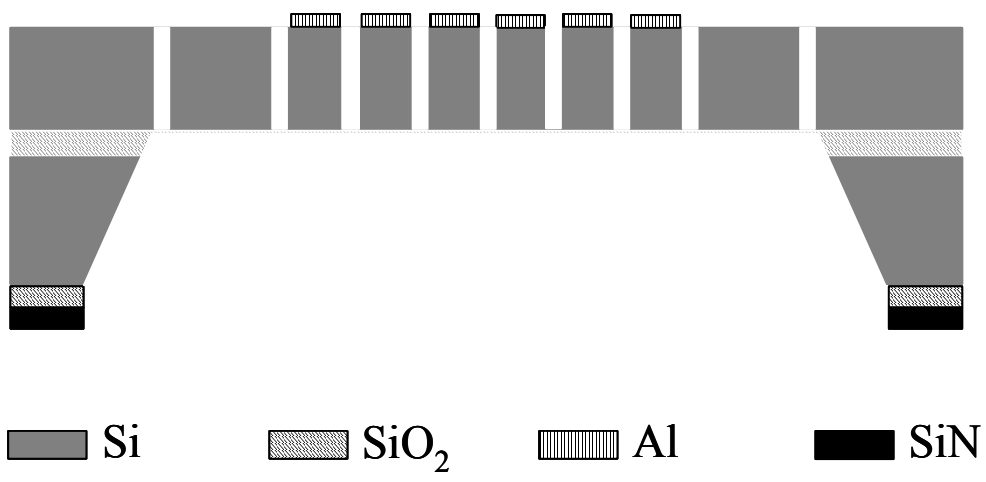

Figure 3: Cross-section of the fabricated device

Due to the process requirements, the device was designed to withstand mechanical shocks as strong as $2000 \mathrm{~g}$. The results of the shock resistance simulations are given in Figure 4.

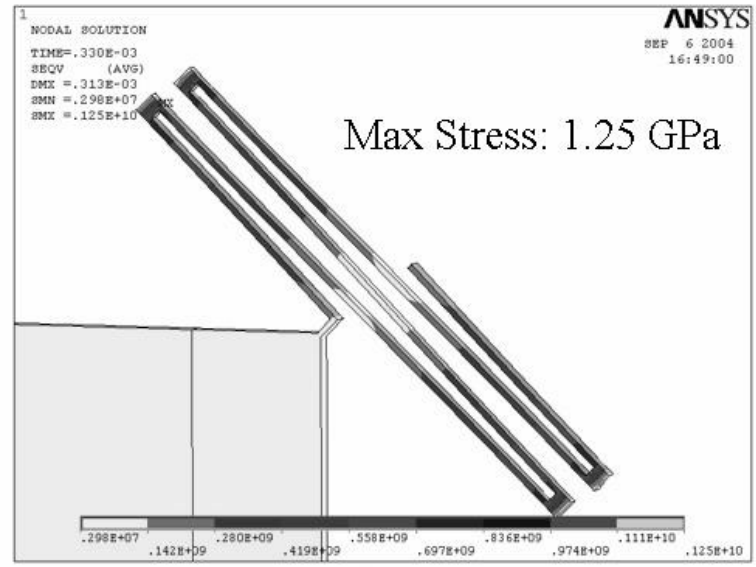

(a)

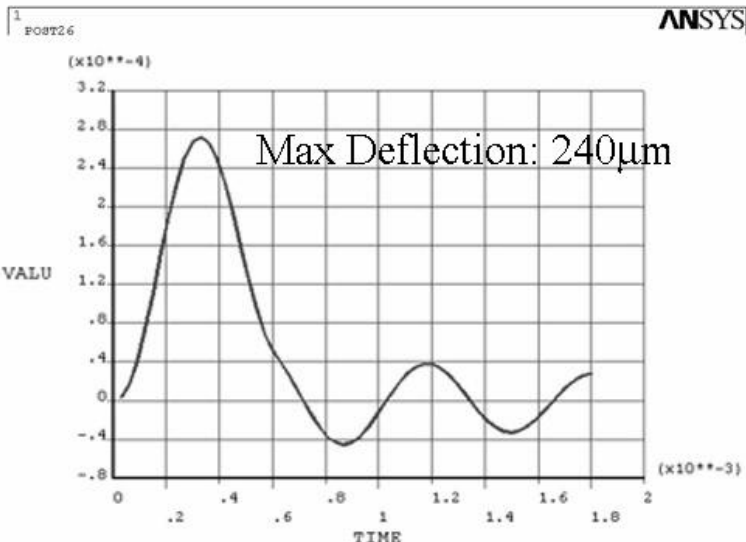

(b)

Figure 4: FEA results showing the shock resistance of the device. A) A $2000 \mathrm{~g}$ shock of $600 \mu$ s duration along the

\section{OPERATION PRINCIPLE OF THE SPECTROMETER}

In its simplest form, a Fourier transform spectrometer consists of two mirrors, one of which can be moved along its normal direction, located perpendicularly to each other and with a beam splitter placed at the vertex of the right angle and oriented at a $45^{\circ}$ angle relative to the two mirrors. This is a Michelson Interferometer configuration with a movable mirror. Radiation incident on the beam splitter from one of the two "ports" is then divided into two parts, each of which propagates down one of the two arms and is reflected off one of the mirrors. The two beams are then recombined and transmitted out the other port. When the position of one mirror is continuously varied along the axis of the corresponding arm, an interferogram is swept out as the two phaseshifted beams interfere with each other [6]. This interferogram encodes the spectrum of the light illuminating the 
mirrors. An LGI uses the same principle, however the splitting of the radiating beam is accomplished by a diffraction grating, and interference of the split waves is achieved by diffraction.

\subsection{Out-of-plane Mode Comb Actuators as Movable Diffraction Gratings}

The grating introduces a rectangular phase profile to the illuminating uniform wavefront, due to the phase difference between the portions of the wavefront reflected from the front and back facets of the grating. The intensity profile of the diffracted light will be diffracted with propagation. Right after the grating, diffracted light has the identical profile as the grating. Far field diffraction pattern of the light will have the shape of the Fourier Transform of the diffraction grating.

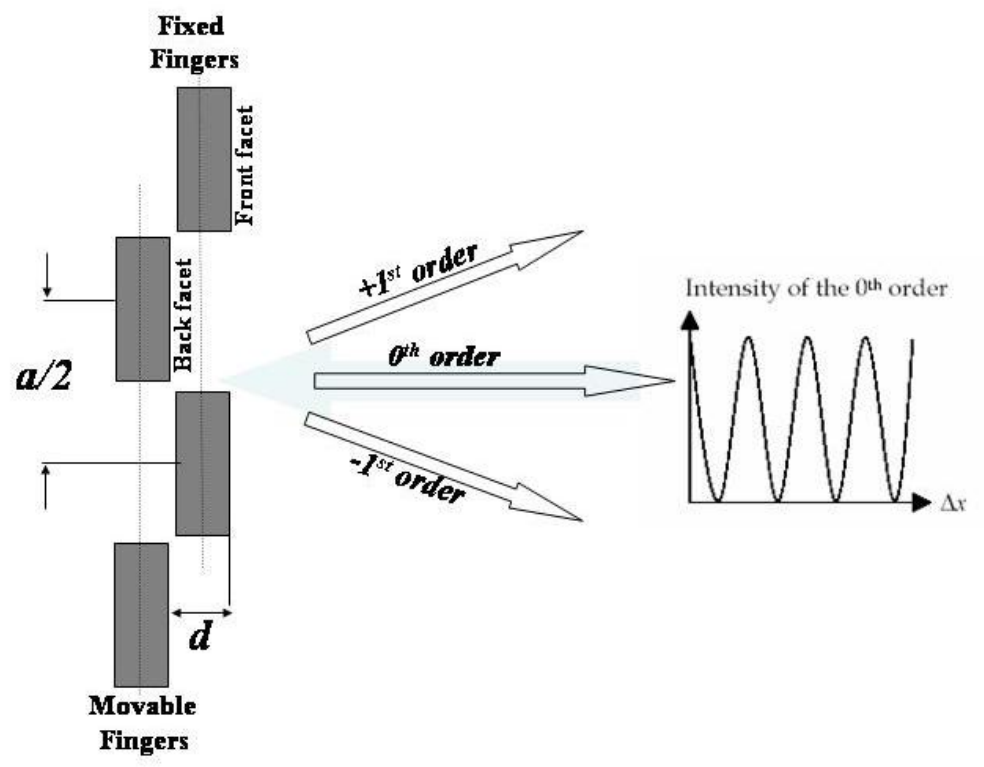

Figure 5: Schematics of a comb-drive rectangular diffraction grating illuminated by monochromatic light, and modulation of the zeroth order intensity as the grating depth $d$ is continuously varied over a distance of multiple wavelengths.

Due to the periodicity of the fingers, comb actuators can be utilized as a tunable diffraction grating, which is a good candidate for Lamellar Grating Interferometry. If a resonating out-of-plane mode comb structure is used as a diffraction grating, the intensity recorded by a detector placed at the center of the diffraction plane would be [2]

$$
I_{\text {center }}=A \cos ^{2}\left(\frac{4 \pi d_{\max } \cos 2 \pi f_{\text {res }} t}{\lambda}\right)
$$

where, $f_{\text {res }}$ is the resonant frequency of the comb structure. Equation 3 implies that at the center of the diffraction plane, the light intensity is sinusoidally modulated with respect to the OPD introduced by the grating. Therefore, while the movable fingers of the spectrometer are resonating, the intensity recorded by a single photodetector placed at the center of the diffraction profile with respect to grating depth will be modulated with a raised cosine function. However, due to the sinusoidal speed variation of the fingers, frequency of the raised cosine intensity modulation will be chirped. This chirp can easily be corrected electronically to produce the desired intensity vs. grating depth signal, which is a raised cosine of period $\lambda / 4$. A Fourier transform operation performed on this signal would directly lead to the wavelength of the illuminating light. The same principle can also be used for spectral analysis of broadband sources. 


\section{THEORETICAL AND EXPERIMENTAL PERFORMANCE ANALYSIS}

\subsection{Mechanical Response}

Fabricated devices were tested under atmospheric pressure, and they were successfully excited in the desired out-of-plane mode. The damped resonance frequency of the structure is measured to $1223 \mathrm{~Hz}$.

Figure 6 shows the measured frequency response of the device. The frequency response of the spectrometer shows a hysteretic behavior. Amplitude of the oscillations makes sudden jumps at two different frequencies. The interval between these two jump frequencies $f 1$ and $f 2$ is the unstable region of the response curve. Oscillations in this region can only be observed if the external frequency is quasi-statically swept down to this region from above. The oscillation frequency of the device is half the excitation frequency, which is called a subharmonic resonance. This type of frequency characteristics indicates that the system is a parametric oscillator. Similar system dynamics are also observed in torsional comb-actuated microscanner devices. Dynamics of such a system were investigated in detail in our earlier works [8].

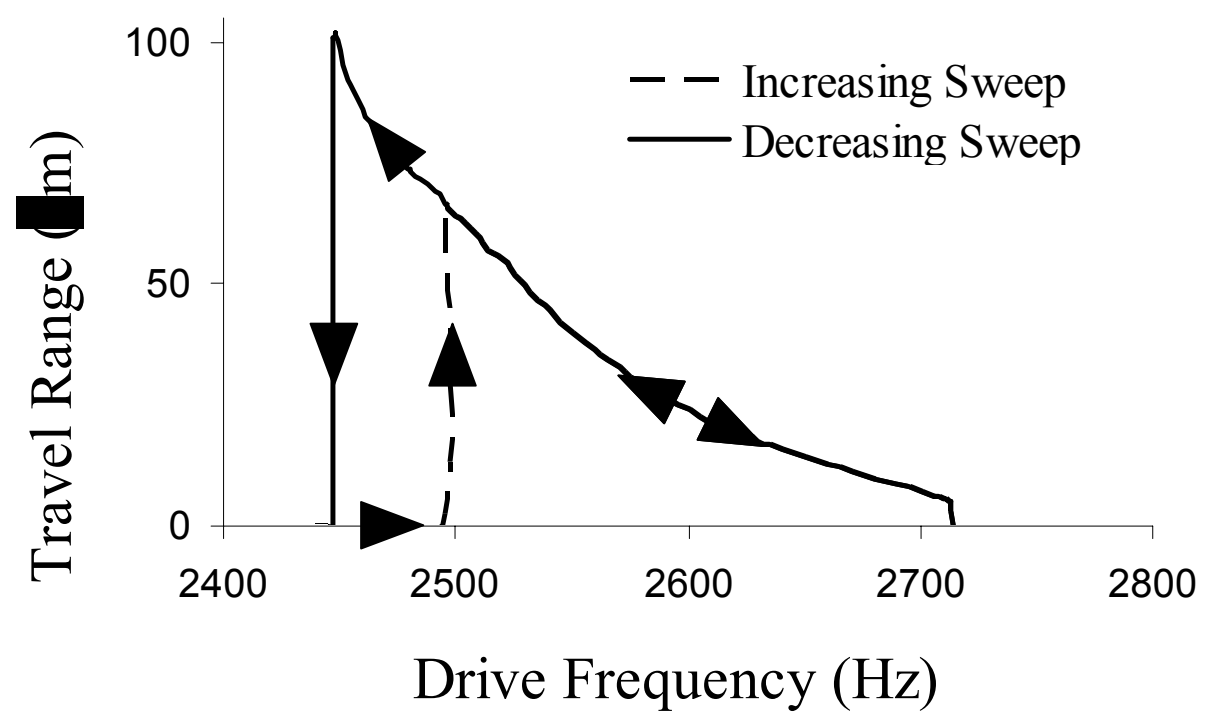

Figure 6: Hysteretic frequency response of the spectrometer with 28V excitation amplitude

\subsection{Spectral Resolution}

As mentioned, the device is capable of producing $\pm 52 \mu \mathrm{m}$ vertical deflection in the resonant mode with $28 \mathrm{~V}$ excitation. This leads to a theoretical spectral resolution of $\sim 0.5 \mathrm{~nm}$ in the visible range and $\sim 5 \mathrm{~nm}$ in the telecom wavelengths. Figure 7 shows the simulation results for spectral resolution of the device. The simulations are performed with MATLAB. 


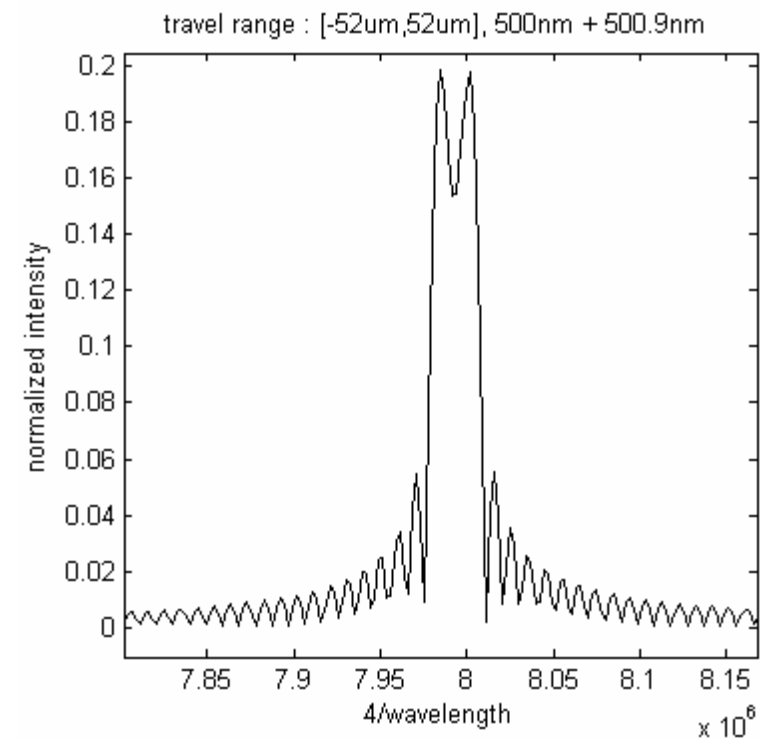

(a)

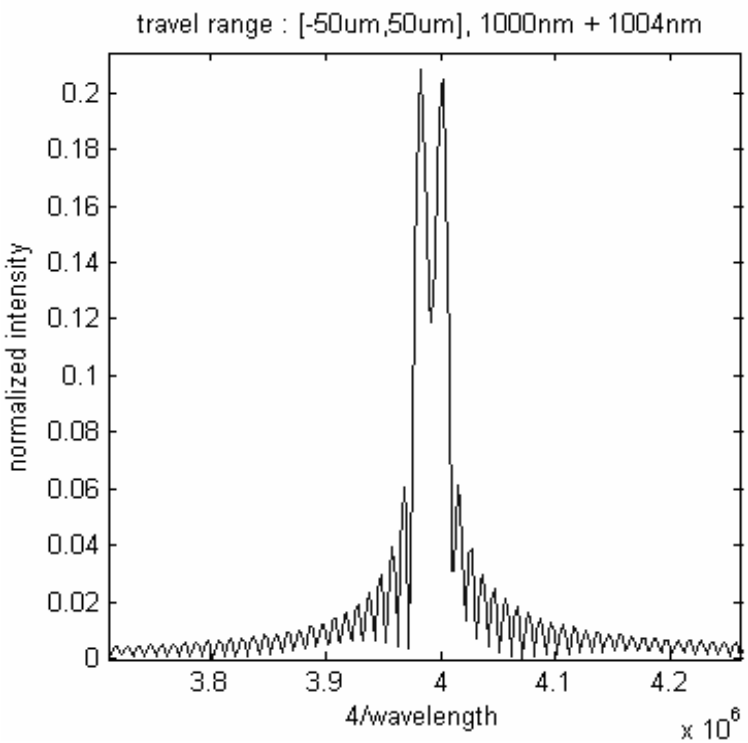

(b)

Figure 7: Spectral resolutions of the specified design simulated using the maximum travel range values obtained from Figure 6. The device provides a resolution of (a) $0.9 \mathrm{~nm}$ at $500 \mathrm{~nm}$, and $3.6 \mathrm{~nm}$ at $1000 \mathrm{~nm}$.

\subsection{Optical Efficiency}

The use of the polished top surface and out-of-plane actuation produces good flatness and a large clear aperture $(3 \mathrm{~mm} \times 3 \mathrm{~mm})$ for the incident beam. Since the structure utilizes the $0^{\text {th }}$ order diffracted light without requiring additional optics or beam splitters, the theoretical optical efficiency is $>80 \%$ and limited only by the fill-factor of the comb fingers. This efficiency level is significantly better than its counterparts.

Experimental diffraction efficiency of the device is measured to be $15 \%$, which is much lower than the theoretical value (Figure 8). This is due to the stress-induced bow of the long and thin comb fingers. Due to this bow, a built-in height difference between the comb fingers are present, and this yields to diffraction, even in the static case. Figure $8 \mathrm{c}$ shows an interferometric picture of the static and movable fingers. The height difference between the fingers is clearly visible from this figure. 


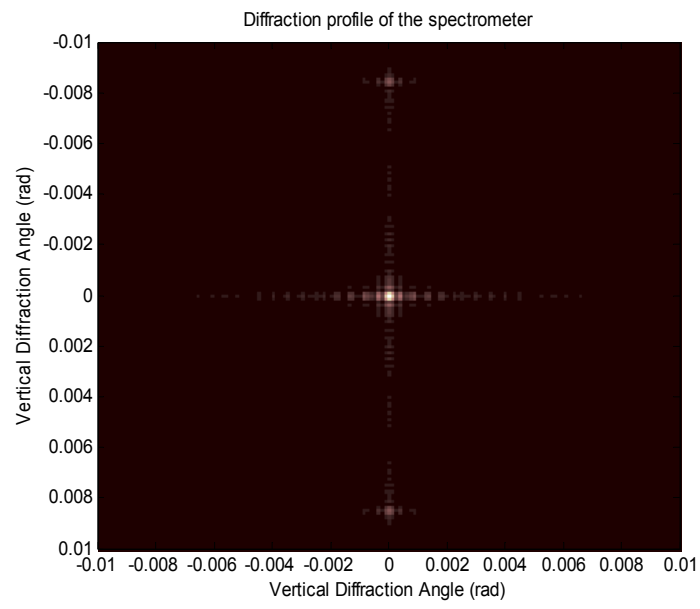

(a)

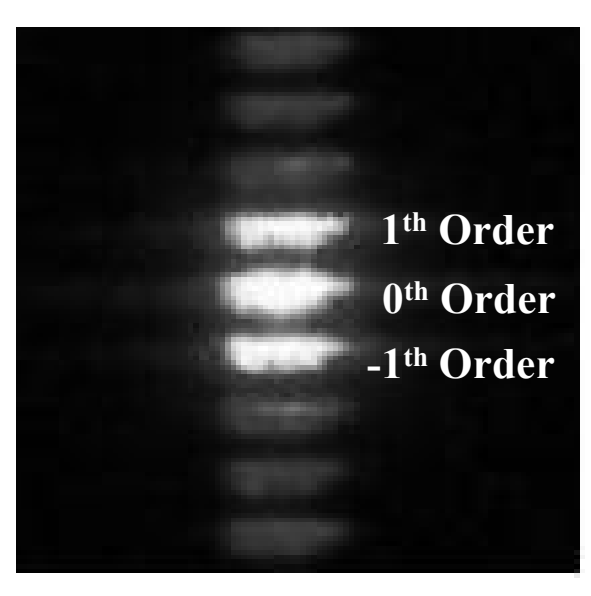

(b)

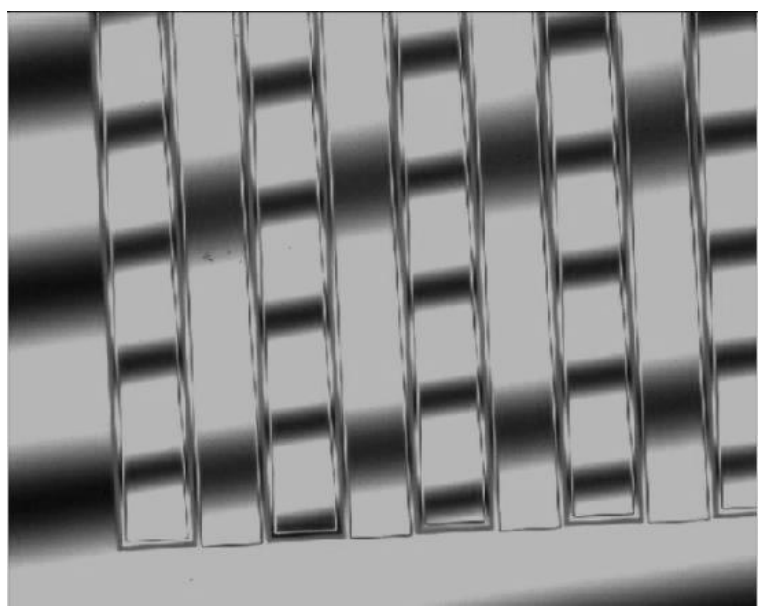

(c)

Figure 8: Diffraction efficiency of the spectrometer system. (a) Diffraction simulation results for zero OPD. Efficiency is \%XX. b) Experimental diffraction profile for zero OPD. Efficiency is \%15, which is significantly lower than the theoretical value. c) White-light Interferometric picture of the movable and fixed fingers that show the bending of the fingers.

\subsection{Spectral Measurements}

Spectrum measurements are performed with a single photodetector placed at $0^{\text {th }}$ order. Due to the resonant motion of the device, the speed of the movable fingers is not constant, but sinusoidally varying. Therefore, the intensity recorded by the detector $(I(t))$ for a monochromatic source is a chirped (i.e. varying frequency) sinusoid. 


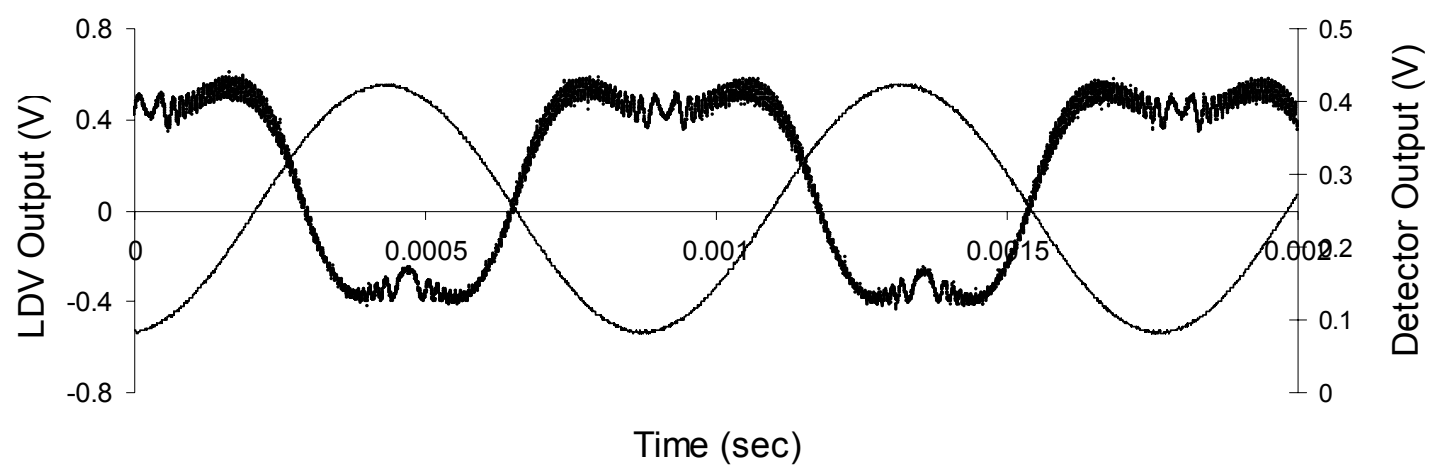

Figure 9: Experimental measurement data on the modulation of 0th order intensity as the movable fingers resonate. The travel range of the comb fingers in this case is $30 \mu \mathrm{m}$

Figure 9 gives the experimental data showing how the $0^{\text {th }}$ order intensity modulated as the movable fingers of the device resonate with a travel range of $30 \mu \mathrm{m}$. The chirped cosine modulation of the intensity can be seen on top of a bigger sinusoidal signal of comb oscillation frequency.

\section{CONCLUSION}

A comb-actuator based Fourier Lamellar Grating Interferometer is designed and implemented. The operation principle of the device is simple and can easily be integrated into a fully functional spectrometer system with a single photodetector and readout electronics. Proposed design can move $100 \mu \mathrm{m}$ along out-of-plane direction which yields a resolution better than $0.5 \mathrm{~nm}$ in the visible band and better than $5 \mathrm{~nm}$ for telecom wavelengths. A thicker SOI wafer can be used to improve the deflection range and the resolution of the spectrometer in future design iterations.

The proposed design has many advantages compared to other spectrometers available in the literature: Fabrication and operation principles of the device are simple. This minimizes the size, complexity, cost and design effort of a fully functional system. Only moving part is the resonating MEMS structure. Small system size enables portable devices. Out-of-plane resonant mode operation of the comb actuators allows for large deflections (high spectral resolution) and high speed while providing a large light collection mirror area. Due to the operation principle, with slight modifications in the device dimensions, spectrometers for very wide range of wavelengths can be realized.

\section{ACKNOWLEDGEMENTS}

The authors would like to thank Dr. Harald Schenk for sharing his experience on microspectrometers, and to Zeljko Skokic for his help on the mask design.

\section{REFERENCES}

[1] P.R. Griffiths, Fourier Transform Infrared Spectrometry, John Wiley \& Sons, 1986.

[2] O. Manzardo, H. P. Herzig, C. R. Marxer, N. F. de Rooij, "Miniaturized time-scanning Fourier transform spectrometer based on silicon technology," Opt. Lett., vol. 24, pp. 1705-1707, 1999.

[3] Kenda, W. Scherf, R. Hauser, H. Grüger, H. Schenk, "A Compact Spectrometer based on a Micromachined Torsional Mirror Device,” Proceedings of IEEE Sensors, vol. 3, pp.1312-1315, 2004.

[4] G. Niederer, H. P. Herzig, M. T. Gale, M. Salt, M. Schnieper, H. Thiele, C. Zschokke, "Resonant Grating Filters at Oblique Incidence", SPIE Proceedings, Vol. 5 183-06,200 
[5] H. Schenk, P. Dürr, T. Haase, D. Kunze, U. Sobe, H. Lakner, H. Kück, "Large deflection micromechanical scanning mirrors for linear scans and pattern generation," Journal of Selected Topics in Quantum Electronics, Vol. 6, No. 5, p.715-722, 2000.

[6] "Fourier Transform Spectrometer," available online at http://scienceworld.wolfram.com/physics/FourierTransformSpectrometer.html

[7] C. Ataman, H. Urey, " Modeling and characterization of comb actuated resonant microscanners, "Journal of Micromechanics and Microengineering, Vol. 16 pp. 9-16, 2006. 